\title{
QUALITY CONTROLLED COMPRESSION OF POLYSOMNOGRAMS
}

\author{
Carl Taswell and Juergen Niederholz \\ Computational Toolsmiths, Stanford, CA 94309-9925
}

\begin{abstract}
A novel compression algorithm incorporating the near-best wavelet packet transform is introduced for multi-modal signals in low bitrate telemonitoring applications. The method permits flexible control of the total bitrate subject to the constraints of the channel, and the allocation of the total bitrate to each of the constituent modes subject to the quality preferences of the observer. Its performance is demonstrated using polysomnograms.

Keywords - near-best bases, wavelet packet transforms, compression, polysomnograms, telemedicine.
\end{abstract}

\section{INTRODUCTION}

Near-best wavelet packet transforms (NB-WPT) [1] provide an effective means for compressing multi-channel multi-modal signals in low bitrate telemonitoring applications [2]. The adaptivity of the method facilitates its use in flexible systems capable of allocating a proportional assignment of the total available bitrate to the separate modes of a multi-modal signal. This capability enables a selective quality-on-demand feature when controlled by a remote observer who adjusts the bitrate allocation by increasing the percentage for the mode of interest and decreasing it for the others. To demonstrate feasibility of a NB-WPT algorithm for quality controlled compression of a multi-modal signal, we study polysomnograms with 4 different signal modes (ECG, EEG, BP, and NT) as an extension of the work in the accompanying paper [2].

\section{Methods}

We considered multi-channel multi-modal signals with $K$ modes and $C_{k}$ channels per mode. All channels were segmented to length $N$ yielding multi-channel data sets with $N \sum_{k=1}^{K} C_{k}$ samples. Each data set was transformed with the NB-WPT using a single decomposition for all channels, and compressed using $M$ transform coefficients with $M<N$. The near-best basis search was performed with a cost function [2] modified using contributions from each mode weighted by the corresponding control parameter $q_{k}$ with $1=\sum_{k=1}^{K} q_{k}$. The $M$ coefficients retained were also allocated accordingly such that there were $M_{k}=q_{k} M$ coefficients with highest absolute value for each mode. Experiments were performed on 5 polysomnograms from the MIT-BIH Database [3] using a total of 760 segments of length $N=64$ and transform parameters as in [2].

\section{Results}

The left plot of Figure 1 displays the rate-distortion curves that resulted for the ECG mode $k=1$ when assigned values of $q_{1}=1,0.75,0.50$, and 0.25 while the other modes were assigned $q_{k}=\left(1-q_{1}\right) / 3$ for $k=2,3,4$. At a total rate of $M=32$ per segment, these values of $q_{1}$

Tel/Fax: 650-323-4336/5779; Email: taswell@toolsmiths.com
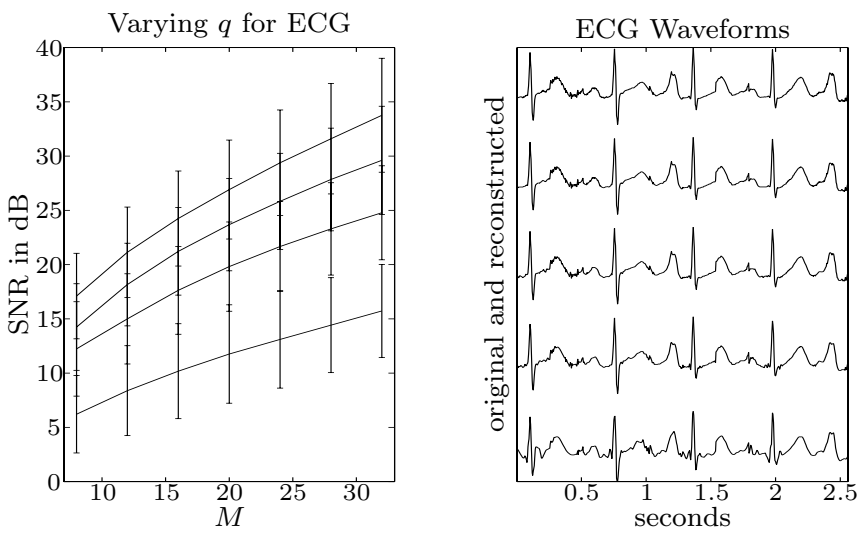

Fig. 1. Left: rate-distortion curves for ECG at $q=1.0,0.75,0.5$, 0.25 ; Right: example segment with normalized waveforms of original and reconstructed ECG at $q=1.0,0.75,0.5,0.25$; both in decreasing order.

correspond to $M_{1}=32,24,16$, and 8 transmitted coefficients for mode $k=1$. The right plot shows an example segment with the original and reconstructed waveforms demonstrating the qualitative results which were obtained with these $q$ values when $M=32$. Analogous results were observed for each of the other signal modes.

\section{Discussion}

Using polysomnograms as a test signal class, we have demonstrated the feasibility of a novel compression algorithm for multi-channel multi-modal signals. The method incorporates an adaptive NB-WPT with a novel modeweighted cost function and a single decomposition per segment for all modes in order to minimize the side information. The method permits extremely flexible control of both the total bitrate subject to the constraints of the transmission channel, and the proportional allocation of the total bitrate to each of the constituent modes subject to the preferences of the remote observer. We are now investigating alternative variations on the basic scheme with comparative evaluations of their performance.

\section{Conclusions}

A new compression algorithm based on the adaptive NB-WPT provides flexible control of compression subject to varying rate and quality demands in low bitrate telemonitoring applications with multi-modal signals.

\section{REFERENCES}

[1] C. Taswell, "Satisficing search algorithms for selecting near-best bases in adaptive tree-structured wavelet transforms," IEEE Trans. Signal Process., pp. 2423-2438, Oct. 1996.

[2] J. Niederholz and C. Taswell, "Near-best WPT compression of polysomnograms," Submitted to BMES-EMBS'99, 1999.

[3] G. Moody, "MIT-BIH polysomnographic database." CD-ROM, August 1992. 